\section{Functions of the Stomach and Intestine}

Edited by M. H. F. Friedman. Pp. xv +469 , hard cover, illustrated. HM + M, Aylesbury, Bucks, 1975. $£ 10.50$.

In such a growing field as the understanding of gastrointestinal physiology and its derangements it is a pleasure to see another book bringing us up to date on normal gastrointestinal function. Such a background is so necessary to the clinician as an insight into the complexity and diffuseness of this branch of gastroenterology. At first sight such a galaxy of notable contributors suggests a rich mine of information, and although this is true, it becomes rapidly apparent that this necessarily applies only to those very specific topics with which individual authors are involved. In other words, the title of the publication rather belies its contents because it does not provide a general coverage of gastrointestinal function, but rather a series of reviews of some realms of current knowledge (remembering that the symposium was held in November 1973) some of which are specialized, such as the essay on factors influencing mesenteric blood flow, and some of which are more general, such as the chapter on the mechanism of the intestinal absorption of sugars and aminoacids. This book, therefore, becomes of prime value to those wishing to acquire a research interest in one of the fields covered, in which case particular articles will provide a good background review, albeit somewhat brief in many cases. In these instances the rather well laid out reference sections at the end of each chapter will be of value.

A section of the book of particular interest is the final one entitled pathologic physiology. The articles within this are comprehensive reviews of the five topics selected but are again quite brief and thus probably of more value to the inquiring postgraduate than the more experienced gastroenterologist.

In short, I would suggest that this book is a worthwhile addition to a gastroenterology library, a slight luxury for a more general medical library, but probably not of lasting value to the individual. The price of the publication somewhat reinforces this.

\section{Recent Advances in Renal Disease}

By N. F. Jones (Ed). Pp. xiii +425, hard cover, illustrated. Edinburgh: Churchill Livingstone, 1975. $£ 11.00$.

This book is a collection of reviews considered by the editor to be 'growing points' in nephrology. Many aspects of the speciality are covered with chapters on physiology, infection, glomerular disease, hypertension, acute and chronic renal failure, and transplantation. The reviews of sodium excretion and antidiuretic hormone are particularly lucid. In many instances, for example the chapters on acute renal failure and renal osteodystrophy, there is a good balance between academic, clinical and therapeutic aspects of the topic but, in spite of three chapters devoted to glomerular disease, drug therapy is not discussed. There is a clear account of what is known about pathogenetic mechanisms in exexperimental and human glomerulonephritis but the important problem of 'chronic glomerulonephritis' is not mentioned. The book will, however, be welcomed by all nephrologists and the editor is to be congratulated for the spectrum of topics included.

\section{Short Practice of Surgery (Bailey \& Love's)}

Revised by A. J. Harding Rains \& H. David Ritchie. Sixteenth edition. Pp. xii +1308 , illustrated, hard cover. London: H. K. Lewis \& Co., Ltd, 1975. £10.00.

The appearance of the sixteenth edition of 'Bailey \& Love' makes it without doubt the most widely read text book of surgery in the world and it has been in continuous use now since 1932. Many novelists would envy its total printing to date of a record 300,000 copies. Italians, Spaniards and Turks can read it in their own languages into which it has been translated. Its success is based on a simple formula of numerous and interesting illustrations, clear and arresting text and lively presentation. There is sadness in the fact that McNeil Love, surely one of the most popular figures in surgical teaching and associated with the book since its first appearance, died before seeing this edition appear.

As well as the two principal authors, there are specialist chapters on orthopaedics by Michael Freeman, neurosurgery by Geoffrey Knight, chest diseases by William Cleland and urology by Peter Philip. A large panel of distinguished experts has also helped with revision of the text and Dr Fairer has brought the useful biographical footnotes up to date. The whole range of general surgery is amply covered and there is adequate consideration of the surgical aspects of the various tropical diseases, which will be of particular value both to overseas readers and to students in this country who are more and more likely to see examples of exotic diseases.

This outstanding book can be recommended without hesitation as the best British undergraduate text in surgery. In addition, it remains the best single volume to cover the topics required by candidates presenting themselves for the Fellowship examinations of the surgical colleges.

\section{A Short Textbook of Haematology}

4th Edition. By R. B. Thompson. Fourth edition. Pp. 387, illustrated, soft cover. Tunbridge Wells, Kent: Pitman Medical, 1975. $£ 5.50$.

The fourth edition of this well known textbook of haematology, which was first published in 1961, follows closely the style and content of the three previous editions. The text consists of a balanced, concise, easy to read review of the common aspects of haematology. It will serve both for undergraduates and as a primer for postgraduates who can use the selected references at the end of each chapter as a guide to further literature.

The main criticism of the book is that it is not sufficiently up-to-date and, partly as a consequence of this, the text is rather dull. A number of signs of age appear. For instance, the statement on page 18 that the MCV and the MCH are of less importance than the MCHC is based on manual cell counting which has largely been abandoned and replaced by electronic cell counting which provides accurate MCV and $\mathrm{MCH}$ measurements which now far exceed the MCHC in practical value. Granulocyte transfusions and bone marrow transplantation receive no mention, nor do the serum ferritin and lysosyme assays. Many well characterized clinical conditions such as pre-leukaemia and smouldering leukaemia, hairy cell leukaemia and chronic myelomonocytic leukaemia do not appear at all. The neurological findings in pernicious anaemia are described at length over four pages, but there is no mention of the vitamin $B_{12}$ binding proteins or methylmalonic aciduria, while unusual but interesting recently recognized congenital defects of vitamin $B_{12}$ or folate metabolism receive either no mention or only a passing reference. Further evidence of lack of up-dating is provided by the only illustrations of response to treatment in acute leukaemia. A child with acute lymphoblastic leukaemia is treated with 6-mercaptopurine and steroids which is not now a drug combination of first choice, while an adult with acute myeloid leukaemia is treated with 6-mercaptopurine alone, whereas most centres now give multiple chemotherapy initially in this disease. 